\title{
Correction to: Methods of Photometric and Radiometric Measurements
}

\section{Correction to:}

Chapter 2 in: M. Bukshtab, Photometry, Radiometry, and Measurements of Optical Losses, Springer Series in Optical Sciences 209, https://doi.org/10.1007/978-981-10-7745-6_2

In the original version of the book, typos have been corrected in Chapter 2 . The book and chapter have been updated with the changes. 